\title{
Protective effects of atorvastatin on cerebral vessel autoregulation in an experimental rabbit model of subarachnoid hemorrhage
}

\author{
JUN-HUI CHEN ${ }^{1 *}$, TING WU $^{2 *}$, LI-KUN YANG $^{1 *}$, LEI CHEN $^{1}$, \\ JIE ZHU ${ }^{1}$, PEI-PEI LI ${ }^{1}, \mathrm{XU} \mathrm{HU}^{1}$ and YU-HAI WANG ${ }^{1}$ \\ Departments of ${ }^{1}$ Neurosurgery and ${ }^{2}$ Cardiology, 10lst Hospital of PLA, Wuxi, Jiangsu 214044, P.R. China
}

Received October 14, 2016; Accepted June 8, 2017

DOI: $10.3892 / \mathrm{mmr} .2017 .8074$

\begin{abstract}
The aim of the present study was to assess the therapeutic effects of atorvastatin on cerebral vessel autoregulation and to explore the underlying mechanisms in a rabbit model of subarachnoid hemorrhage (SAH). A total of 48 healthy male New Zealand rabbits (weight, $2-2.5 \mathrm{~kg}$ ) were randomly allocated into SAH, Sham or SAH + atorvastatin groups ( $n=16 /$ group). The Sham group received $20 \mathrm{mg} / \mathrm{kg} / \mathrm{d}$ saline solution, whereas $20 \mathrm{mg} / \mathrm{kg} / \mathrm{d}$ atorvastatin was administered to rabbits in the $\mathrm{SAH}+$ atorvastatin group following $\mathrm{SAH}$ induction. Changes in diameter, perimeter and basilar artery (BA) area were assessed and expression levels of the vasoactive molecules endothelin-1 (ET-1), von Willebrand factor (vWF) and thrombomodulin (TM) were measured. Neuronal apoptosis was analyzed $72 \mathrm{~h}$ following SAH by terminal deoxynucleotidyl-transferase-mediated dUTP nick-end labeling (TUNEL) staining. The mortality rate in the SAH group was $18.75,25 \%$ in the $\mathrm{SAH}+$ atorvastatin treated group and $0 \%$ in the Sham group ( $n=16 /$ group). The neurological score in the $\mathrm{SAH}+$ atorvastatin group was $1.75 \pm 0.68$, which was significantly higher compared with the Sham group $(0.38 \pm 0.49$; $\mathrm{P}<0.05)$. The $\mathrm{BA}$ area in the $\mathrm{SAH}+$ atorvastatin group (89.6 \pm 9.11$)$ was significantly lower compared with the SAH group $(115.4 \pm 11.0 ; \mathrm{P}<0.01)$. The present study demonstrated that SAH induction resulted in a significant increase in the diameter, perimeter and cross-sectional area of the BA in the $\mathrm{SAH}+$ atorvastatin group. Administration of atorvastatin may significantly downregulate the expression levels of ET-1, vWF and TM (all $\mathrm{P}<0.01$ ) vs. sham and SAH groups. TUNEL staining demonstrated that neuronal apoptosis was remarkably reduced in the hippocampus of SAH rabbits following treatment with atorvastatin $(\mathrm{P}<0.05)$. Atorvastatin treatment may alleviate cerebral vasospasm and mediate structural and
\end{abstract}

Correspondence to: Dr Yu-Hai Wang, Department of Neurosurgery, 101st Hospital of PLA, 101 Xing Yuan North Road, Wuxi, Jiangsu 214044, P.R. China

E-mail: wangyuhai67@126.com

*Contributed equally

Key words: subarachnoid hemorrhage, atorvastatin, autoregulation functional remodeling of vascular endothelial cells, in addition to promoting anti-apoptotic signaling. These results provided supporting evidence for the use of atorvastatin as an effective and well-tolerated treatment for $\mathrm{SAH}$ in various clinical settings and may protect the autoregulation of cerebral vessels.

\section{Introduction}

Spontaneous subarachnoid hemorrhage (SAH) is a common cerebral vascular condition that affects 9 out of 100,000 people in the Western world $(1,2)$. Although it accounts for $~ 5 \%$ of all strokes, it is a devastating neurological condition with poor outcomes and high mortality and morbidity (1-3). Early brain injury (EBI), acute hydrocephalus, delayed cerebral vasospasm (CVS) and cerebral infarction are important factors that may contribute to poor outcome post-SAH. However, data from a previous study indicated that treatment of CVS, using targeted medication in patients with $\mathrm{SAH}$, may not lead to improved neurological outcomes, and suggested that CVS may not be the only cause of the delayed ischemic complications (4). Additional studies reported that the functional outcome did not improved with reversal of angiographic vasospasm (5), and that treatment with an endothelin receptor antagonist may significantly improve CVS post-SAH (6), but it did not improve the poor outcome.

Cerebral vessel autoregulation is the intrinsic ability of the cerebral vasculature to maintain stable blood flow even during changes in blood pressure, such as cerebral perfusion pressure (7). A recent review of the clinical effects of disturbed cerebral vessel autoregulation following aneurysmal SAH aimed to determine whether patients with SAH may benefit from therapeutic targeting of cerebral vessel autoregulation (4). However, large-scale analyses are required to determine clinical benefits, and it was suggested that statins may serve an important role in cerebral vessel autoregulation.

Statins, which are inhibitors of the 3-hydroxy-3-methylglutaryl-coenzyme A (HMG-CoA) reductase, are widely used in cardiovascular therapy as cholesterol-lowering drugs that may have pleiotropic effects, including anti-inflammatory (8), antioxidative stress $(9)$, anti-CVS $(10,11)$ and inhibition of platelet aggregation $(12,13)$. Atorvastatin is a potent statin that inhibits HMG-CoA reductase; however, the specific pathophysiological mechanisms of statins on cerebral vessel autoregulation and EBI following SAH remain unknown. Therefore, the aim of 
the present study was to evaluate the effects of atorvastatin on SAH-induced EBI, CVS and function of vascular endothelial cells. Results revealed that atorvastatin was an effective and well-tolerated treatment for $\mathrm{SAH}$ in various clinical settings and may protect the autoregulation of cerebral vessels. The present study investigated whether clinical benefits could be achieved by influencing the state of cerebral vessels autoregulation.

\section{Materials and methods}

Animals and drugs. Animal's care and experimental protocols were approved by the Animal Care and Use Committee of Anhui Medical University (Hefei, China) and conformed to the Guide for the Care and Use of Laboratory Animals as outlined by the National Institutes of Health. New Zealand white rabbits used in the present study were purchased from Animal Central of TaiHu Hospital (cat. no. SYXK2012-0033; Wuxi, China). Rabbits were raised in a comfortable room with normal atmospheric moisture and fed with a standard diet every 3-4 h at the Animal Center of Taihu Hospital for 10 days prior to the start of the experiments. The temperature of the feeding and operation rooms was maintained at $22^{\circ} \mathrm{C}$. Atorvastatin (20 mg/kg/d; Pfizer, Inc., Wuxi, China) was administered by gastric gavage once daily for 3 days prior to SAH operation and $22 \mathrm{~h}$ post-SAH to maintain drug levels (14). Neurological deficits were assessed $24 \mathrm{~h}$ following SAH operation and rabbits were euthanized immediately following evaluation. The data of blood pressure, injected arterial blood gas data, body weight and blood pressure were used for monitoring only, and were not shown in the results of the present study.

Experimental design. A total of 48 adult male New Zealand white rabbits (weight, 2.5-3.2 kg) were randomly assigned to 3 groups ( $n=16 /$ group): i) Sham group, which received an injection of saline into the cisterna magna $(2 \mathrm{ml})$; ii) $\mathrm{SAH}$ group; and iii) $\mathrm{SAH}+$ atorvastatin group. As 3 rabbits died in the SAH group and 4 rabbits died in the $\mathrm{SAH}+$ atorvastatin group, 3 additional rabbits were added for the $\mathrm{SAH}$ group and 4 additional rabbits were added for the $\mathrm{SAH}+$ atorvastatin group. Atorvastatin $\left(20 \mathrm{mg} / \mathrm{kg}^{\prime} \mathrm{d}\right)$ was administered when the SAH model was established and continued every 24 for $72 \mathrm{~h}$, as previously described (10). A total of 8 rabbits from each group were sacrificed on day 3 with the fixation-perfusion method in $10 \%$ formaldehyde. The hippocampus and basilar artery (BA) were removed for terminal deoxynucleotidyl-transferase-mediated dUTP nick-end labeling (TUNEL), hematoxylin and eosin (H\&E) and for immunohistochemical staining analyses. The remaining eight rabbits were exsanguinated and decollated, and the artery was removed and frozen in deep cryogenic freezer for further biochemical studies. Prior to sacrifice, serum samples were collected from each rabbit to evaluate the protein expression levels of endothelin-1 (ET-1) by ELISA.

SAH model rabbit establishment. SAH was induced according to a previously described two-hemorrhage rabbit model $(10,14)$. Briefly, rabbits were anesthetized with an auricular marginal vein injection of $10 \%$ chloral hydrate $(2.5 \mathrm{ml} / \mathrm{kg})$. Vital signs were kept stable and a 23-gauge butterfly needle was inserted into the cisterna magna. When cerebrospinal fluid was observed from the butterfly needle, then $2 \mathrm{ml}$ non-heparinized fresh autologous auricular arteries blood was injected into the cisterna magna from the butterfly needle with strict aseptic technique and the injection lasted $1 \mathrm{~min}$. To maintain the blood flow from cisterna magna to the basilar cistern, all rabbits were held at a 30-degree head-down position for $30 \mathrm{~min}$. Rabbits were returned to the feeding room following recovery from anesthesia. The second injection was administered after $48 \mathrm{~h}$ in a similar manner.

Neurological scoring. Neurological scores were recorded by the same independent observer who was blinded to the study. A previously modified scoring table was used to evaluate the neurological function every day $(14,15)$. The Neurological study consisted of the following three tests: i) Appetite. Scores indicate the following: 2, Scarcely ate; 1, left meal unfinished; 0, finished meal. ii) Activity. Scores indicate the following: 2, Almost always lying down; 1, lying down, will stand and walk with some stimulation; 0, active, alert or standing. iii) Deficits. Scores indicate the following: 2, Impossible to walk and stand due to ataxia and paresis; 1 , unable walk due to ataxia or paresis; 0 , no deficits.

Fixation-perfusion. A total of 8 rabbits from each group were anesthetized with an auricular marginal vein injection of $10 \%$ chloral hydrate $(4 \mathrm{ml} / \mathrm{kg})$. The chest was quickly cut open for cannula intubation in the left ventricle and the right atrium was cut open. Perfusion started with $1,500 \mathrm{ml}$ of physiological phosphate buffered saline (PBS; $\mathrm{pH} \mathrm{7.3)}$ at $37^{\circ} \mathrm{C}$, followed by $1,000 \mathrm{ml}$ buffered formaldehyde $(10 \%)$ under $120 \mathrm{~cm} \mathrm{H}_{2} \mathrm{O}$ perfusion pressure. Following fixation-perfusion, whole brain tissues were removed and stored in $10 \%$ formalin at $22^{\circ} \mathrm{C}$.

$H \& E$ staining. This tissue was then post-fixed in $4 \%$ paraformaldehyde at $4^{\circ} \mathrm{C}$ overnight, dehydrated and embedded in paraffin, and cut into sections $(4 \mu \mathrm{m})$. To avoid the arterial branches, the BAs were transected at the same middle position each time $(2 \mathrm{~mm})$. Every fourth section along the coronal plane was stained with H\&E and observed under a microscope. The diameter, perimeter and cross-sectional area of the BA were measured by an independent investigator, blinded to the study, using an Olympus light microscope (Olympus Corporation, Tokyo, Japan) and Image-Pro Plus 6.0 Software (Media Cybernetics, Inc., Rockville, MD, USA). The diameter, perimeter and cross-sectional area of BA were calculated as previously described (16). T-score indicates the BA perimeter/BA thickness.

Immunohistochemical staining. Brain tissues (brainstem) were isolated from brain tissues before storing in formalin, and were routinely fixed, embedded and cut into sections $(4 \mu \mathrm{m})$ for immunohistochemistry. Endogenous peroxidase was quenched with $3 \%$ hydrogen peroxide for $10 \mathrm{~min}$ at room temperature. Sections were incubated with anti-von Willebrand factor (vWF; cat no. ab778; dilution 1:30; Abcam, Cambridge, UK) and anti-thrombomodulin (TM; cat no. ab6980; 1:1,000; Abcam) primary antibodies overnight at $4^{\circ} \mathrm{C}$, followed by incubation with HRP-conjugated secondary antibodies (goat serum; cat no. ab138478; Abcam) for $1 \mathrm{~h}$ at room temperature. Following 
washing, sections were developed in 3,3'-diaminobenzidine (DAB) solution and counterstained with hematoxylin for $5 \mathrm{~min}$ at room temperature. Negative controls were included by omitting the primary antibody. A light Olympus microscope (Olympus Corporation) and Image-Pro Plus 6.0 software (Media Cybernetics, Inc.) were used for analysis.

TUNEL staining and cell counting. The TUNEL Staining kit (Roche Diagnostics, Basel, Switzerland) was used for hippocampus staining, and the TUNEL-positive cells were analyzed by fluorescein-dUTP with dNTP or POD with DAB (manufacturer's protocol for in situ apoptosis detection kit (Roche Diagnostics) according to the methods described previously (17). A negative control was used by eliminating the TUNEL reaction mixture. Cells exhibiting nuclear condensation/fragmentation and apoptotic bodies in the absence of cytoplasmic TUNEL reactivity, brown staining of nuclei were considered as apoptotic cells. Apoptotic cells were confirmed with the help of a pathologist blinded to the grouping. The number of TUNEL-positive cells in each region (number $/ \mathrm{mm}^{2}$ ) were counted in a high-powered field (magnification, $x 400$ ) by an investigator who was blinded to the study. A total of 8 rabbits from each group were used. A total of 5 fields were analyzed, and the experiment was repeated three times.

Western blot analysis. Western blot analysis was performed as described previously for evaluating the levels of Caspase-3 proteins (18). The samples (20 $\mu \mathrm{g}$ protein), as determined by using a bicinchoninic acid assay (Abcam), were separated by $10 \%$ SDS-PAGE and transferred to a nitrocellulose membrane. Membranes were probed with the following primary antibodies: Rabbit anti-Caspase-3 (cat no. ab4051; 1:500; Abcam) antibody. GAPDH (cat no. G5262; 1:6,000; Sigma-Aldrich; Merck KGaA, Darmstadt, Germany) was used as a loading control. Following incubation with the primary antibodies for $1 \mathrm{~h}$ at room temperature, membranes were washed with TBS $+5 \%$ Tween-20 (TBST) and incubated with appropriate horseradish peroxidase-labeled secondary antibodies (cat no. sc2357; 1:1,000; Santa Cruz Biotechnology, Inc., Dallas, TX, USA) for $1 \mathrm{~h}$ at room temperature in $1 \%$ nonfat milk in TBST for $1 \mathrm{~h}$ at room temperature. Following two rinses and four washes with PBST, membranes were incubated with Enhanced Chemiluminescence Western Blotting Detection Reagent (GE Healthcare Life Sciences, Shanghai, China) for $60 \mathrm{sec}$ and exposed to autoradiography film for visualization of the bands. Results were quantified by Quantity One version 4.5 software (Bio-Rad Laboratories, Hercules, CA, USA). A total of 8 rabbits from each group were used.

ELISA. At day 3 following surgical intervention, blood samples were collected from anesthetized animals ( $n=8 /$ group) and analyzed for ET-1 expression levels using a rabbit ET-1 ELISA kit (cat no. F2003; Westang Bio-Tech Co., Ltd., Shanghai, China) specific for rabbits. Plasma was separated from the blood by centrifugation at 3,000 $\mathrm{x} g$ for $15 \mathrm{~min}$, and the supernatant was assayed for the protein concentrations of ET-1, according to the manufacturer's protocol. ET-1 concentrations $(\mathrm{pg} / \mathrm{ml})$ were determined based on a standard curve, prepared using a known set of serial dilutions of standard proteins. The experiment was repeated three times.
Reverse transcription-quantitative polymerase chain reaction (RT-qPCR). Total RNA was extracted from hippocampus brain samples ( $n=8 /$ group) using TRIzol Reagent (Gibco; Thermo Fisher Scientific, Inc., Waltham, MA, USA), following the manufacture's protocol. $\beta$-actin was used as an internal control. First-strand cDNA was synthesized from the total RNA as previously described by using a kit from Abcam (cat no. 185916) $(10,19)$. vWF and TM mRNA levels in each sample were determined by qPCR using SYBR Green Master Mix (Toyobo Co., Ltd., Osaka, Japan). The qPCR thermocycling conditions were as follows: $45^{\circ} \mathrm{C}(2 \mathrm{~min})$ and $95^{\circ} \mathrm{C}$ (10 min), followed by 40 cycles of denaturation $95^{\circ} \mathrm{C}(15 \mathrm{sec})$; annealing $60^{\circ} \mathrm{C}(1 \mathrm{~min})$; extension $72^{\circ} \mathrm{C}(1 \mathrm{~min})$. All samples were analyzed in triplicate. The primers were as follows: TM, forward CTCTAGCACCTACAATACCCCATT, reverse CCC GAGTCCAGTGTCCCTCT (146); vWF, forward TTTTCT TATGTTCTCCACGAAGGG, reverse CATTGATGAGGC AGGGGTTCT (151); $\beta$-actin, forward CCCATCTATGAG GGTTACGC, reverse TTTAATGTCACGCACGATTTC (150) (20).

Statistical analysis. All data were presented as the mean \pm standard deviation. SPSS 14.0 statistical software (SPSS, Inc., Chicago, IL, USA) was used for statistical analysis. Differences between the two groups were analyzed using a two-tailed unpaired Student's t-test. The differences among multiple groups were assessed using a one-way analysis of variance followed by Tukey's post hoc test. Ranked data between the two groups were evaluated using rank sum test. $\mathrm{P}<0.05$ was considered to indicate a statistically significant difference.

\section{Results}

General observations. No significant differences were identified in blood pressure, injected arterial blood gas data, body weight and blood pressure (data not shown). The mortality of SAH group was $18.75 \%$ (3 of 16), 25\% (4 of 16) in SAH + atorvastatin group and $0 \%$ in the Sham group. No significant difference was identified for the mortality of SAH + atorvastatin treated group compared with the SAH group $(\mathrm{P}>0.05)$. During the process of model establishment, the dead and not eligible rabbits were excluded from the present study and were replaced with new rabbits to maintain the number of animals in each group.

Neurological scoring. The neurological scores of rabbits in $\mathrm{SAH}+$ atorvastatin group were significantly lower than the scores in the SAH group at $72 \mathrm{~h}$ following $\mathrm{SAH}$ induction $(\mathrm{P}<0.05$; Fig. 1; Table I). In addition, treatment with atorvastatin was demonstrated to improve neurological functional post-SAH in experimental rabbits.

$H \&$ E staining for morphometric vasospasm. Notable differences were observed in BA morphology among the three groups on day 3 post-SAH (Fig. 2A): The inner perimeter, diameter and cross-sectional area of BA in the SAH group was smaller, and the BA wall was thicker compared with the Sham group. To avoid the individual differences, the relatively fixed values of $\mathrm{T}(\mathrm{T}=$ inner perimeter/wall thickness) were calculated 
Table I. Neurological scores among the three experimental groups.

\begin{tabular}{lcc}
\hline Group & $\mathrm{n}$ & Score \\
\hline Sham & 16 & $0.38+0.19$ \\
SAH & 16 & $2.25+0.39$ \\
SAH + atorvastatin & 16 & $1.75+0.28$ \\
\hline
\end{tabular}

SAH, subarachnoid hemorrhage.

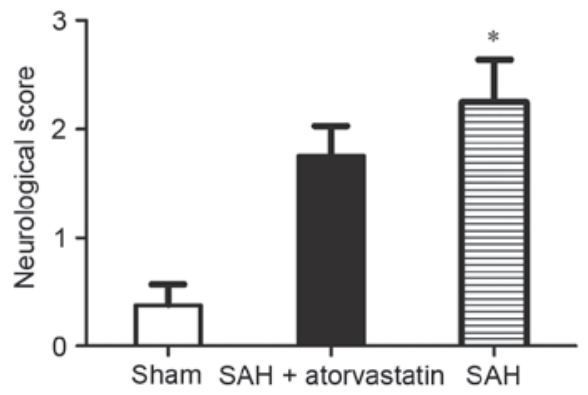

Figure 1. Neurological scores in the experimental groups following SAH. The results indicated that the neurological score was significantly higher in $\mathrm{SAH}$ rats compared with rats in the Sham group at $72 \mathrm{~h}$ Atorvastatin reduced the neurological scores significantly than $\mathrm{SAH}$ group. ${ }^{\text {"P }}<0.05 \mathrm{SAH}+$ atorvastatin vs. SAH group. SAH, subarachnoid hemorrhage.

(Fig. 2B). Compared with the SAH group, the T value of the $\mathrm{BA}$ in the $\mathrm{SAH}+$ atorvastatin treatment group exhibited a significant increase $(115.4 \pm 11.0$ vs. $89.6 \pm 9.11 ; \mathrm{P}<0.01)$.

Immunohistochemical staining for $v W F$ and TM. Immunohistochemical analysis revealed only a few vWF- and TM-positive regions located in the vessels in the Sham group (Fig. 3A). However, vWF and TM expression levels were upregulated in the $\mathrm{SAH}$ and $\mathrm{SAH}+$ atorvastatin treatment groups. The $\mathrm{SAH}$ group and the $\mathrm{SAH}+$ atorvastatin treatment groups exhibited a significant increase in the number of vWFand TM-positive immunostained regions compared with the Sham group $(\mathrm{P}<0.01$; Fig $3 \mathrm{~B})$. Immunohistochemical analysis also indicated a significant decrease in the expression vWF and $\mathrm{TM}$ in the $\mathrm{SAH}+$ atorvastatin treatment group compared with the SAH group $(\mathrm{P}<0.01)$.

TUNEL staining and cell counting for apoptosis. Few TUNEL-positive cells were detected in the hippocampus of rabbits in the Sham group. However, TUNEL-positive cells were significantly increased in SAH-induced rabbits at $72 \mathrm{~h}$, and TUNEL-positive cells were significantly decreased in the $\mathrm{SAH}+$ atorvastatin treatment group compared with untreated SAH rabbits $(\mathrm{P}<0.05$; Fig. 4$)$.

ELISA for ET-1 protein expression. ELISA was performed to examine the alterations in protein concentration of the vasoconstrictor ET-1 to determine the effects of atorvastatin on cerebral edema post-SAH. Compared with the Sham group,
ET-1 expression was significantly upregulated in the SAH rabbits $(85.24+6.25$ vs. $39.72+4.67 \mathrm{pg} / \mathrm{ml}$; P<0.01; Fig. 5). Following atorvastatin treatment, the elevation of plasma ET-1 concentration was significantly lower compared with the SAH group (62.92+7.27 vs. 85.24+6.25 pg/ml; P<0.01; Fig. 5).

$R T$-qPCR for mRNA of $v W F$ and TM. vWF and TM mRNA expression levels of were detected by RT-qPCR: vWF and TM mRNAs were expressed at low levels in the brain tissue in the Sham group, whereas the levels of vWF and TM mRNA expression were significantly increased in the SAH and $\mathrm{SAH}+$ atorvastatin treatment groups. Compared with the SAH group, the vWF and TM mRNA expression levels were reduced in the $\mathrm{SAH}+$ atorvastatin treatment group. $(\mathrm{P}<0.01$; Fig. 6).

Caspase-3 protein expression. Caspase-3 protein expression in the hippocampus was detected by western blot to observe neuronal apoptosis at $72 \mathrm{~h}$ following SAH. SAH induced an evident increase of Caspase-3 expression levels in the hippocampus compared with the Sham group, whereas the level of Caspase- 3 was markedly decreased in the $\mathrm{SAH}+$ atorvastatin group ( $\mathrm{P}<0.05$ vs. SAH; Fig. 7).

\section{Discussion}

To the best of our knowledge, the present study is the first to demonstrate the therapeutic effects of atorvastatin on early brain injury, CVS and cerebral vessels autoregulation following experimental SAH in rabbits. CVS was evaluated by examination and quantification of BA morphology, cerebral vessel autoregulation by evaluating the expression levels of vWF and TM, and early brain injury was examined by TUNEL staining.

Several previous studies have demonstrated that statins may significantly inhibit the activity of matrix metalloproteinase (MMP)-2 and MMP-9 to maintain the stability of the blood/brain barrier (BBB) $(17,21)$. Another study reported that pitavastatin exerted its neuroprotective effects by inhibiting the activation of c-Jun N-terminal kinase p46/p55 and reducing the expression levels of cleaved Caspase-9a and MMP-9 (22). Simvastatin has also been demonstrated to protect the cerebrum from neuronal excitotoxicity and cytotoxic edema by reducing the expression of phosphorylated Calcium/calmodulin-dependent protein kinase type II and AQP4 in experimental animals with ischemic stroke (18). Tseng et al (11) were the first to demonstrate by a phase II randomized placebo-controlled trial that acute treatment with pravastatin following SAH was safe and was able to improve CVS and cerebral autoregulation, and reduce vasospasm-related delayed ischemic deficits, and the poor prognostic rate was reduced. One other study reported that simvastatin was safe for the prevention of delayed cerebral ischemia following $\mathrm{SAH}$ in a randomized placebo-controlled trial with 39 patients (23). The present study demonstrated that atorvastatin may ameliorate early CVS and may protect cerebral vascular endothelial cells in SAH model rabbits, suggesting its potential as a treatment strategy to reverse CVS and EBI in patients suffering from SAH.

However, previous studies $(24,25)$ have reported contradictory results in which statins were not indicated to have a significant impact on brain edema, delayed cerebral ischemia 
A

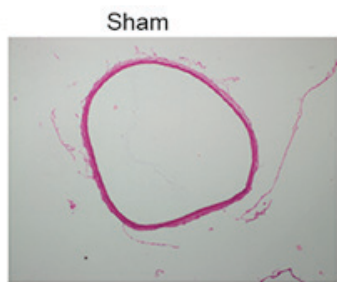

B

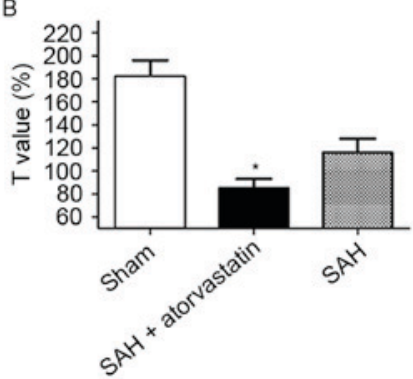

$\mathrm{SAH}+$ atorvastatin
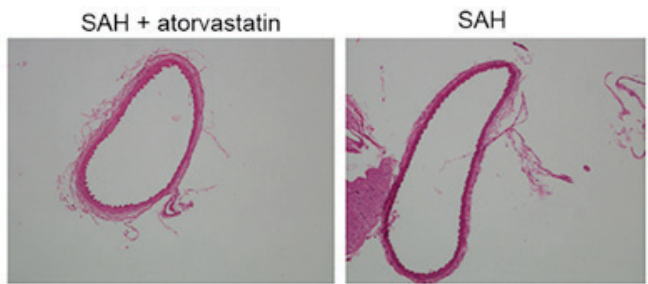

Figure 2. Effects of atorvastatin on the morphology of the BA (T score) in SAH rats. (A) Representative bright field images of the hematoxylin and eosin stained BA in the three experimental groups, procured with a light microscope; magnification, $\mathrm{x} 50$. Diameter and cross-sectional area of the BA in the SAH group was smaller, whereas the BA wall became thicker compared with the Sham group. (B) Comparison of T values for each group. * $<<0.01$ vs. SAH. BA, basilar artery; SAH, subarachnoid hemorrhage.

A

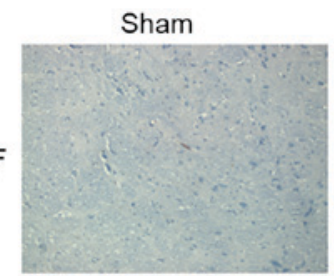

TM

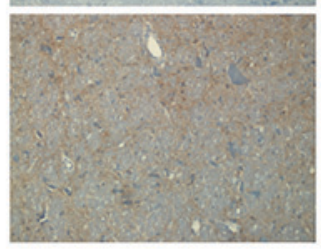

SAH
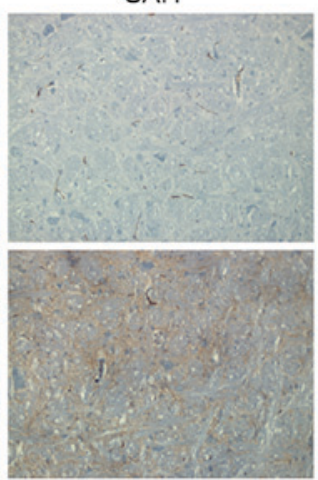

$\mathrm{SAH}+$ atorvastatin

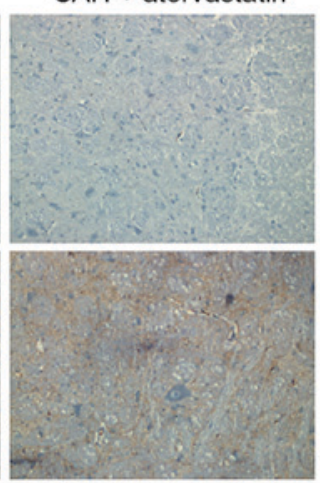

B

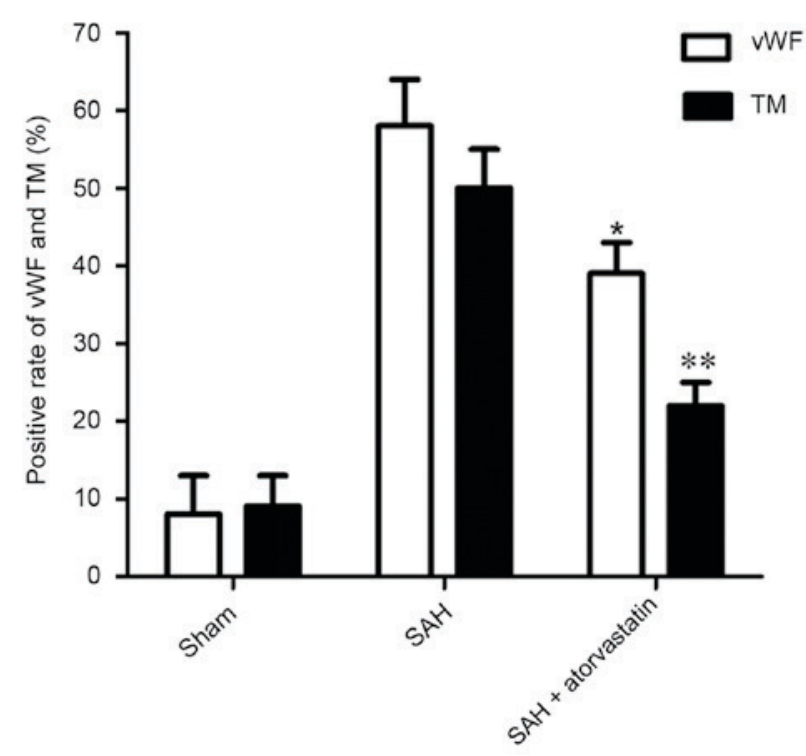

Figure 3. Immunohistochemical staining of brainstem for vWF and TM post-SAH. (A) Few vWF- and TM-positive regions were observed in the vessels in the Sham group, whereas positive staining increased in SAH-induced rats. The SAH-induced increases in expressions were notably reduced by atorvastatin treatment. (B) Quantitative analysis demonstrated a significant reduction in the percentage of positive-stained regions in the SAH + atorvastatin group compared with the SAH group. Magnification, $x 50$. Data are presented as the mean \pm standard deviation; ${ }^{*} \mathrm{P}<0.01$ and ${ }^{* *} \mathrm{P}<0.01$ vs. SAH group. SAH, subarachnoid hemorrhage; TM, thrombomodulin; vWF, von Willebrand factor. 
A
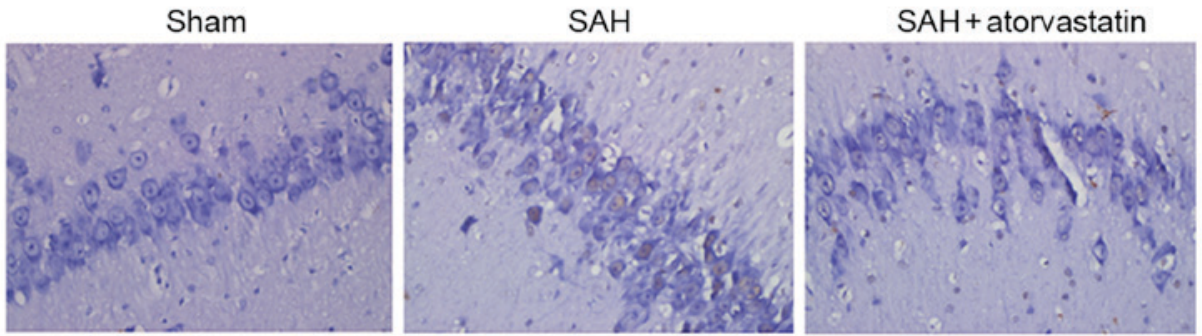

B

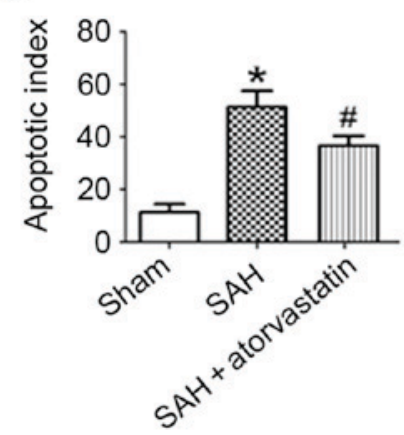

Figure 4. TUNEL staining of hippocampus following SAH induction. (A) Few apoptotic cells were observed in Sham group rabbits. TUNEL-positive staining was markedly increased in SAH rabbits, which was notably reduced by atorvastatin treatment. (B) Quantitative analysis demonstrated a significant increase in the number of apoptotic cells (number $/ \mathrm{mm}^{2}$ ) by SAH induction compared with the number of apoptotic cells in the Sham group; "P<0.05. A significant reduction in number of apoptotic-positive cells was observed in the $\mathrm{SAH}+$ atorvastatin group compared with the SAH group; ${ }^{\#} \mathrm{P}<0.05$. SAH, subarachnoid hemorrhage; TUNEL, terminal deoxynucleotidyl-transferase-mediated dUTP nick-end labeling.

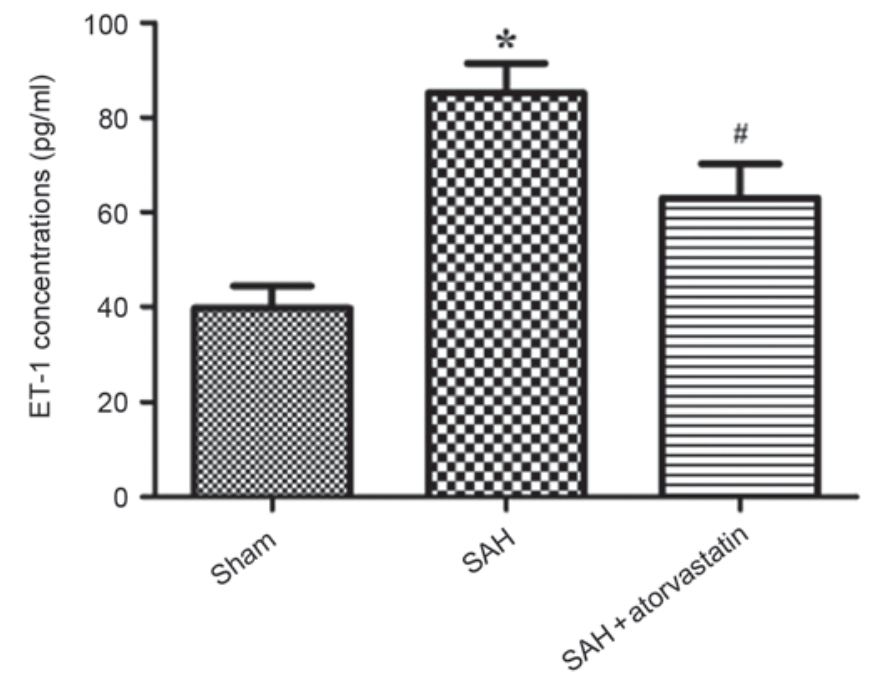

Figure 5. ELISA-based examination of the effects of atorvastatin treatment on the concentration of ET-1 in the plasma of the rabbits at $72 \mathrm{~h}$ following SAH. Graph demonstrating the protein concentration of ET-1 in the three groups ( $n=8 /$ group). Data are presented as the mean \pm standard deviation. ET-1 was significantly increased in SAH group compared with Sham group at $72 \mathrm{~h} ;{ }^{*} \mathrm{P}<0.01$. Atorvastatin treatment significantly decreased the SAH-induced ET-1 expression compared with the SAH group; "P<0.01. ET-1, endothelin 1; SAH, subarachnoid hemorrhage.

and brain injury post-SAH. A multicenter, randomized, controlled, double-blinded clinical trial demonstrated that high- or low-dose simvastatin treatment exerted no long-term effects in the incidence of delayed ischemic deficits or in the rate of favorable outcomes following SAH (24). Another study reported a similar conclusion that no benefit was detected in the use of simvastatin for long-term or short-term outcome in

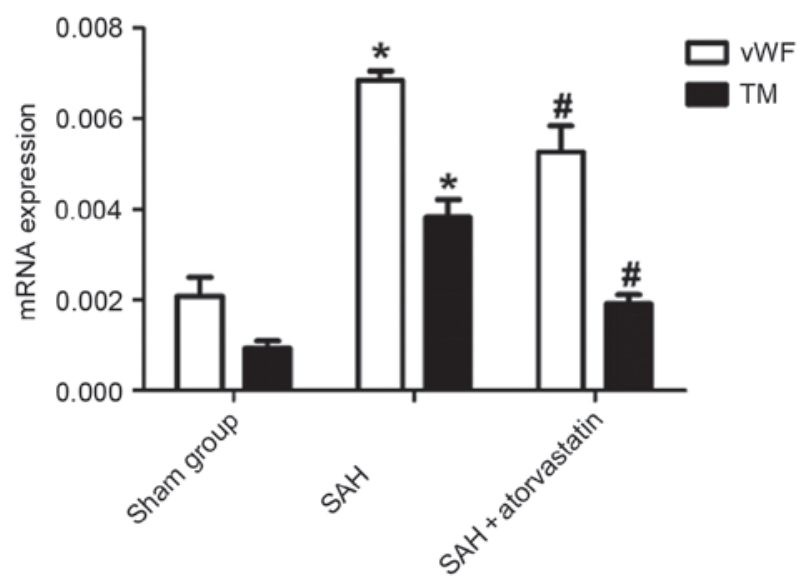

Figure 6. mRNA expression levels of vWF and TM in brain tissues were detected by reverse transcription-quantitative polymerase chain reaction. Data are presented as the mean \pm standard deviation; $n=8 /$ group. The mRNA expression levels of vWF and TM were significantly increased in SAH group compared with the Sham group at $72 \mathrm{~h}$ in the plasma; ${ }^{*} \mathrm{P}<0.01$; Atorvastatin treatment significantly decreased the mRNA expression levels of vWF and TM compared with the $\mathrm{SAH}$ group; ${ }^{\text {P }}<0.01$. SAH, subarachnoid hemorrhage; TM, thrombomodulin; vWF, von Willebrand factor.

patients with SAH in a famous Simvastatin in Aneurysmal SAH trial (25). The present study, however, demonstrated that atorvastatin may have a vasodilatory effect on the intracranial vessels of BA in a rabbit SAH model, indicating its potential as a treatment strategy to alleviate CVS in patients suffering from SAH.

Atorvastatin is an inhibitor of the HMG-CoA reductase, and has been widely used in cardiovascular medicine as a cholesterol-lowering drug. A previous study reported that 
A

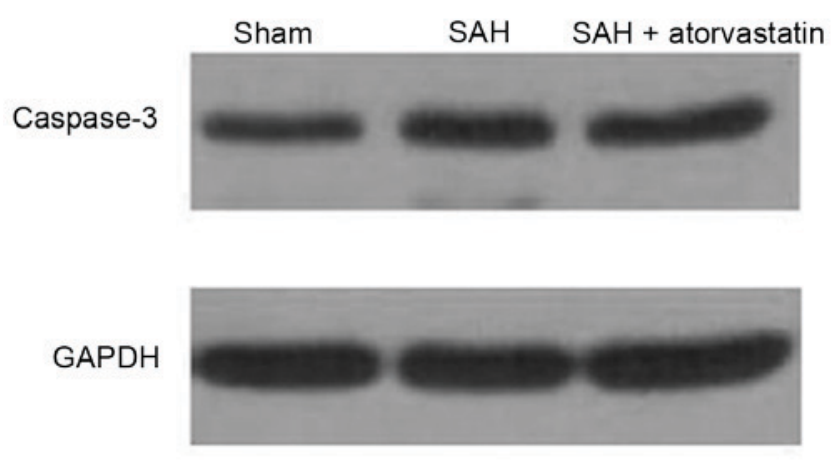

B

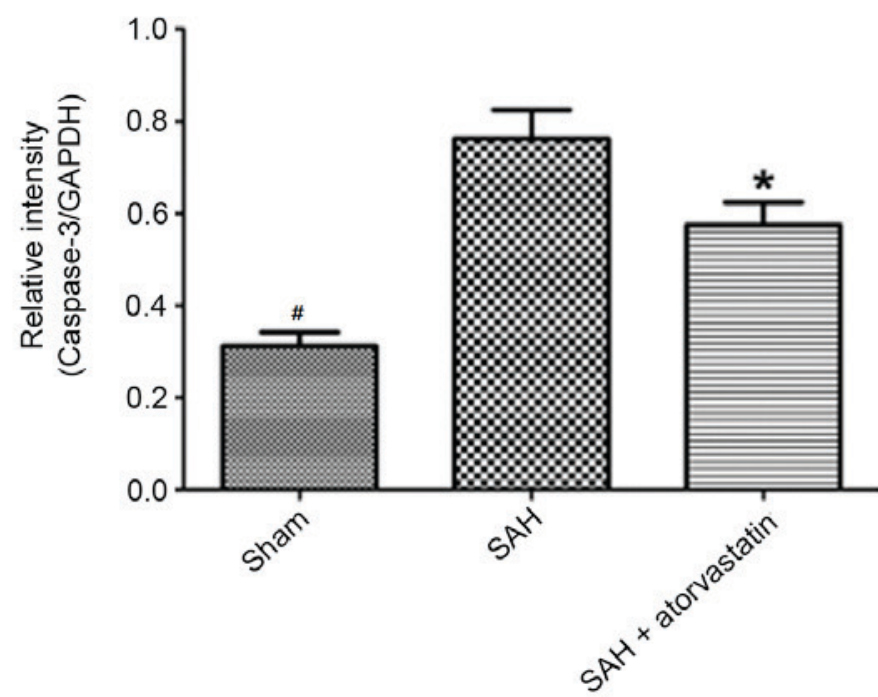

Figure 7. Caspase-3 protein expression in the hippocampus. (A) Representative western blot of Caspase-3 protein expression. (B) Densitometric analysis of Caspase-3 protein expression. Data are presented as the mean \pm standard deviation. Caspase-3 protein expression in the Sham group was significantly lower than $\mathrm{SAH}$ group and $\mathrm{SAH}+$ atorvastatin group $\left(\mathrm{n}=8 /\right.$ group,$\left.{ }^{*} \mathrm{P}<0.01\right)$. Caspase-3 expression levels were significantly decreased in the $\mathrm{SAH}+$ atorvastatin group vs. the SAH group; " $\mathrm{P}<0.05$. Equal protein loading was confirmed by intracellular GAPDH protein expression. SAH, subarachnoid hemorrhage.

atorvastatin was a neuroprotective drug that was able to preserve BBB permeability, decrease brain edema, increase neurological scores and ameliorate CVS, and that atorvastatin may function by inhibiting the Caspase-dependent proapoptotic pathway (10). Atorvastatin was previously demonstrated to reduce the level of ET-1 expression, which corresponded to its antivasospastic effects in chronic vasospasm following SAH-induced vasospasm (26).

A mechanism underlying the anti-CVS or neuroprotective functions of atorvastatin, whether or not a critical reduction in cerebral blood flow has occurred, may be explained by the subsequent constriction of intracerebral vessels, good condition of vascular endothelial cell function and improvement of cerebral blood flow. However, a specific molecular mechanism remains unclear.

To explore the molecular mechanisms underlying atorvastatin function, the expression of three major factors need to be studied as follows: the function of vascular endothelial cell, cerebral vessels autoregulation and contribution to CVS post-SAH. All of them were potent vasoactive peptides synthesized and released by the vascular endothelium and were markers of endothelial function. vWF is a macromolecule glycoprotein with adhesive functions that is mainly stored in platelet activation-dependent granules and Weibel-Palade bodies of the vascular endothelial cells. It is involved with platelet adhesion to the extracellular matrix of vascular endothelial cells and promotes platelet aggregation (27). TM is a single-strand glycoprotein that is also synthesized and stored in vascular endothelial cells; it has anticoagulation effects by combining with thrombin and converts Protein $\mathrm{C}$ into activated protein $\mathrm{C}$ (27). ET-1 has been demonstrated to bind to specific receptors on smooth muscle cells and leads to the constriction of blood vessels and the proliferation of endothelial cells (28), and has deleterious effects on water homeostasis, cerebral edema and BBB integrity. In normal physiological conditions, the expression of these cytokines was minimal. When organism was stimulated severely or the function of cerebral endothelial cells autoregulation suffered damage, the expression of vWF, TM and ET-1 will increase. Therefore, vWF, TM and ET-1 were considered as the 'gold standard' to evaluate the function of cerebral endothelial cells and the cerebral vessel autoregulation $(27,29)$. In the previous study, endothelins was recognized as potent vasoconstrictors, promitogens and inflammatory mediators in the pathogenesis of vasospasm after SAH, it may be critical in the pathogenesis of CVS $(26,30,31)$. Tang et al (32) also found that the expression of vWF was significantly increased at CVS group than the no-CVS group and control group by clinical research. Xu et al (33) demonstrated that TM had protective effects in preserving microvascular integrity after SAH through preserving endothelial junction proteins and quenching apoptosis/inflammation in endothelial cells, and the underlying mechanism may be via blocking of the p38MAPK-p53/NF-кB(p65) pathway. Su et al (34) also found TM analog solution promotes reperfusion and reduces infarct volume in a thrombotic model of stroke.

The present study demonstrated that atorvastatin treatment significantly decreased the expression of $\mathrm{vWF}, \mathrm{TM}$ and ET-1 following SAH induction. These results suggested that atorvastatin may alleviate CVS by regulating the expression of vWF, TM and ET-1. The results indicated that the function of vascular endothelial cell was destroyed and it also led to dysfunction of cerebral autoregulation, the other side. Therefore, this finding suggested that atorvastatin may alleviate CVS by regulating the plasma concentrations of ET-1, $\mathrm{vWF}$ and TM in the clinic. A precise mechanism of action for atorvastatin remains to be elucidated.

Neuronal apoptosis serves an important role of mechanism about delayed neurologic deficits after SAH. Previous studies have demonstrated that p53 also serves an important role in apoptotic cell death following experimental $\mathrm{SAH}$ 
induction $(10,35)$. The present study performed TUNEL analysis to identify and quantify the number of apoptotic cells in the hippocampus of SAH model rabbits treated with and without atorvastatin. Caspases are a family of cysteine proteases that serve essential roles in apoptosis $(10,14,36)$. Caspases-3 is a member of the caspase family and serves an important role in the execution-phase of apoptosis. The expression of the Caspase-3 was reported to be increased in brain neurons following SAH and may promote neuron apoptosis $(3,10)$. The present study demonstrated that the number of TUNEL-positive cells was significantly increased in SAH rabbits, but was significantly decreased in the $\mathrm{SAH}+$ atorvastatin group. These results suggested that atorvastatin treatment may preclude CVS and improve circulation of the brain, and may have neuroprotective effects.

In conclusion, results from the present study suggested that atorvastatin may be used for the treatment of CVS, thus protecting vascular endothelial cell function and maintaining cerebral vessel autoregulation. It is a relatively convenient and affordable method for SAH and may be used in widespread clinical practice. The increased expression of ET-1, vWF and TM may be partly responsible for the therapeutic effects of atorvastatin; atorvastatin treatment may reduce neuronal apoptosis and may be another method for the treatment of SAH and improved outcome. However, the precise mechanism of action of atorvastatin remains to be investigated.

\section{References}

1. Komotar RJ, Schmidt JM, Starke RM, Claassen J, Wartenberg KE, Lee K, Badjatia N, Connolly ES Jr and Mayer SA: Resuscitation and critical care of poor-grade subarachnoid hemorrhage. Neurosurgery 64: 397-411, 2009.

2. Rosengart AJ, Schultheiss KE, Tolentino J and Macdonald RL: Prognostic factors for outcome in patients with aneurysmal subarachnoid hemorrhage. Stroke 38: 2315-2321, 2007.

3. Steiner T, Juvela S, Unterberg A, Jung C, Forsting $M$ and Rinkel G; European Stroke Organization: European Stroke Organization guidelines for the management of intracranial aneurysms and subarachnoid haemorrhage. Cerebrovasc Dis 35: 93-112, 2013

4. Budohoski KP, Czosnyka M, Kirkpatrick PJ, Smirlewski P, Steiner LA and Pickard JD: Clinical relevance of cerebral autoregulation following subarachnoid haemorrhage. Nat Rev Neurol 9: 152-163, 2013.

5. Laskowitz DT and Kolls BJ: Neuroprotection in subarachnoid hemorrhage. Stroke 41 (10 Suppl): S79-S84, 2010.

6. Macdonald RL, Higashida RT, Keller E, Mayer SA, Molyneux A, Raabe A, Vajkoczy P, Wangke I, Bach D, Frey A, et al: Clazosentan, an endothelin receptor antagonist, in patients with aneurysmal subarachnoid haemorrhage undergoing surgical clipping: A randomised, double-blind, placebo-controlled phase 3 trail (CONSCIOUS-2). Lancet Neurol 10: 618-625, 2011.

7. Kontos HA, Wei EP, Navari RM, Levasseur JE, Rosenblum WI and Patterson JL Jr: Responses of cerebral arteries and arterioles to acute hypotension and hypertension. Am J Physiol 234: H371-H383, 1978.

8. Sehba FA, Pluta RM and Zhang JH: Metamorphosis of subarachnoid hemorrhage research: From delayed vasospasm to early brain injury. Mol Neurobiol 43: 27-40, 2011.

9. Tousoulis D, Antoniades C, Katsi V, Bosinakou E, Kotsopoulou M, Tsioufis C and Stefanadis C: The impact of early administration of low-dose atorvastatin treatment on inflammatory process, in patients with unstable angina and low cholesterol level. Int J Cardiol 109: 48-52, 2006.

10. Cheng G, Wei L, Zhi-Dan S, Shi-Guang Z and Xiang-Zhen L: Atorvastatin ameliorates cerebral vasospasm and early brain injury after subarachnoid hemorrhage and inhibits caspase-dependent apoptosis pathway. BMC Neurosci 10: 7, 2009.
11. Tseng MY, Czonsnyka M, Richards H, Pickard JD and Kirkpatrick PJ: Effects of acute treatment with pravastatin on cerebral vasospasm, autoregulation and delayed ischemic deficits after aneurismal subarachnoid hemorrhage: A phase 2 randomized placebo-controlled trial. Stroke 36: 1627-1632, 2005.

12. Lee YM, Chen WF, Chou DS, Jayakumar T, Hou SY, Lee JJ, Hsiao G and Sheu JR: Cyclic nucleotides and mitogen-activated protein kinase: Regulation of simvastatin in platelet. J Biomed Sci 17: 45, 2010.

13. Luzak B, Boncler M, Rywaniak J, Wilk R, Stanczyk L, Czyz M, Rysz J and Watala C: The effect of a platelet cholesterol modulation on the acetylsalicylic acid-mediated blood platelet inhibition in hypercholesterolemic patients. Eur J Pharmacol 658: 91-97, 2011.

14. Chen JH, Yang LK, Chen L, Wang YH, Wu Y, Jiang BJ, Zhu J and Li PP: Atorvastatin ameliorates early brain injury after subarachnoid hemorrhage via inhibition of AQP4 expression in rabbits. Int J Mol Med 37: 1059-1066, 2016.

15. Zhou C, Yamaguchi M, Kusaka G, Schonholz C, Nanda A and Zhang JH: Caspase inhibitors prevent endothelial apoptosis and cerebral vasospasm in dog model of experimental subarachnoid hemorrhage. J Cerebral Blood Flow Metab 24: 419-431, 2004.

16. Hu N, Wu Y, Chen BZ, Han JF and Zhou MT: Protective effect of stellate ganglion block on delayed cerebral vasospasm in an experimental rat model of subarachnoid hemorrhage. Brain Res 1585: 63-71, 2014.

17. Seo JH, Guo S, Lok J, Navaratna D, Whalen MJ, Kim KW and Lo EH: Neurovascular matrix metalloproteinases and the blood-brain barrier. Curr Pharm Des 18: 3645-3648, 2012.

18. Zhu MX, Lu C, Xia CM, Qiao ZW and Zhu DN: Simvastatin pretreatment protects cerebrum from neuronal injury by decreasing the expressions of phosphor-CaMK II and AQP4 in ischemic stroke rats. J Mol Neurosci 54: 591-601, 2014.

19. Koyama Y, Maebara Y, Hayashi M, Nagae R, Tokuyama S and Michinaga S: Endothelins reciprocally regulate VEGF-A and angiopoietin-1 production in cultured rat astrocytes: Implications on astrocytic proliferation. Glia 60: 1954-1963, 2012.

20. Livak KJ and Schmittgen TD: Analysis of relative gene expression data using real-time quantitative PCR and the 2(-Delta Delta C(T)) method. Methods 25: 402-408, 2001.

21. Rosenberg GA and Navratil M: Metalloproteinase inhibition blocks edema in intracerebral hemorrhage in the rat. Neurolgy 48: 921-926, 1997.

22. Chang CZ, Wu SC, Kwan AL and Lin CL: Preconditioning with pitavastatin, an HMG-CoA reductase inhibitor, attenuates C-Jun $\mathrm{N}$-terminal kinase activation in experimental subarachnoid hemorrhage-induced apoptosis. Acta Neurochir (Wien) 157: 1031-1041,2015.

23. Chou SH, Smith EE, Badjatia N, Nogueira RG, Sims JR II, Ogilvy CS, Rordorf GA and Ayata C: A randomized, double-blind, placebo-controlled pilot study of simvastatin in aneurysmal subarachnoid hemorrhage. Stroke 39: 2891-2893, 2008.

24. Wong GK, Chan DY, Siu DY, Zee BC, Poon WS, Chan MT, Gin T and Leung M; HDS-SAH Investigators: High-dose simvastatin for aneurysmal subarachnoid hemorrhage: Multicenter randomized controlled double-blinded clinical trial. Stroke 46: 382-388, 2015.

25. Kirkpatrick PJ, Turner CL, Smith $\mathrm{C}$ and Hutchinson PJ, Murray GD; STASH Collaborators: Simvastatin in aneurysmal subarachnoid haemorrhage (STASH): A multicentre randomised phase 3 trial. Lancet Neurol 13: 666-675, 2014.

26. Chang CZ, Wu SC, Lin CL, Hwang SL, Howng SL and Kwan AL: Atorvastatin preconditioning attenuates the production of endothelin-1 and prevents experimental vasospasm in rats. Acta Neurochir (Wien) 152: 1399-1406, 2010.

27. Blann AD and Tabemer DA: A reliable marker of endothelial cell dysfunction: Does it exist. Br J Haemataol 90: 224-228, 1995.

28. Chow M, Dumont AS and Kasselletal NF: Endothelin receptor antagonists and cerebral vasospasm: An update. Neurosurgery 51: 1333-1342, 2002.

29. Califano F, Giovanniello T, Pantone P, Campana E, Parlapiano C, Alegiani F, Vincentelli GM and Turchetti P: Clinical importance of thrombomodulin serum levels. Eur RevMed Pharmacol Sci 4: 59-66, 2000.

30. Kästner S, Oertel MF, Scharbrodt W, Krause M, Böker DK and Deinsberger W: Endothelin-1 in plasma, cisternal CSF and microdialysate following aneurysmal SAH. Acta Neurochir (Wien) 147: 1271-1279, 2005. 
31. Juvela S: Plasma endothelin concentrations after aneurysmal subarachnoid hemorrhage. J Neurosurg 92: 390-400, 2000.

32. Tang QF, Lu SQ, Zhao YM and Qian JX: The changes of von willebrand factor/a disintegrin-like and metalloprotease with thrombospondin type I repeats-13 balance in aneurysmal subarachnoid hemorrhage. Int J Clin Exp Med 8: 1342-1348, 2015.

33. Xu T, Zhang WG, Sun J, Zhang Y, Lu JF, Han HB, Zhou CM and Yan JH: Protective effects of thrombomodulin on microvascular permeability after subarachnoid hemorrhage in mouse model. Neuroscience 299: 18-27, 2015.

34. Su EJ, Geyer M, Wahl M, Mann K, Ginsburg D, Brohmann H, Petersen KU and Lawrence DA: The thrombomodulin analog Solulin promotes reperfusion and reduces infarct volume in a thrombotic stroke model. J Thromb Haemost 9: 1174-1182, 2011.
35. Cahill J, Calvert JW, Marcantonio S and Zhang JH: p53 may play an orchestrating role in apoptotic cell death after experimental subarachnoid hemorrhage. Neurosurgery 60: 531-545, 2007.

36. Alnemri ES, Livingston DJ, Nicholson DW, Salvesen G, Thomberry NA, Wong WW and Yuan J: Human ICE/CED-3 protease nomenclature. Cell 87: 171, 1996.

This work is licensed under a Creative Commons Attribution-NonCommercial-NoDerivatives 4.0 International (CC BY-NC-ND 4.0) License. 